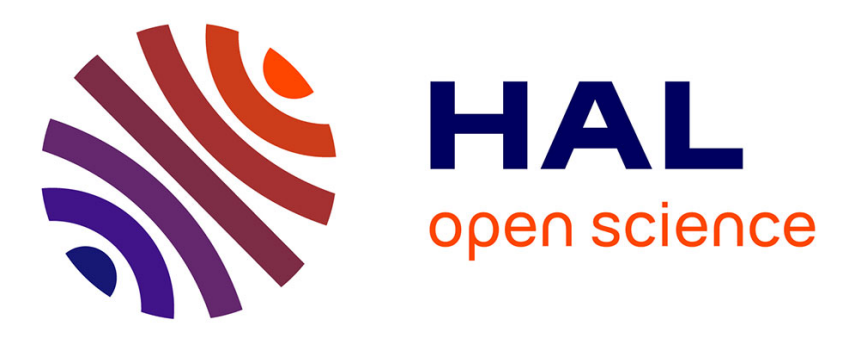

\title{
Adaptation de la viticulture argentine à la variabilité climatique : une approche par simulation dans la région de Mendoza
}

Cyril Tissot, Hervé Quénol, Mathias Rouan

\section{- To cite this version:}

Cyril Tissot, Hervé Quénol, Mathias Rouan. Adaptation de la viticulture argentine à la variabilité climatique : une approche par simulation dans la région de Mendoza. Norois, 2020, 254, pp.91-108. 10.4000/norois.9668 . hal-03093980

\section{HAL Id: hal-03093980 https://hal.science/hal-03093980}

Submitted on 4 Jan 2021

HAL is a multi-disciplinary open access archive for the deposit and dissemination of scientific research documents, whether they are published or not. The documents may come from teaching and research institutions in France or abroad, or from public or private research centers.
L'archive ouverte pluridisciplinaire HAL, est destinée au dépôt et à la diffusion de documents scientifiques de niveau recherche, publiés ou non, émanant des établissements d'enseignement et de recherche français ou étrangers, des laboratoires publics ou privés. 


\title{
Adaptation de la viticulture argentine à la variabilité climatique : une approche par simulation dans la région de Mendoza
}

\section{Adaptation of Argentina viticulture to climate variability: simulation approach in the Mendoza area}

\author{
Cyril Tissot $^{1 *}$, Hervé Quenol ${ }^{2}$, Mathias Rouan ${ }^{1}$ \\ ${ }^{1}$ UMR 6554 CNRS LETG/Brest, cyril.tissot@univ-brest.fr \\ ${ }^{2}$ UMR 6554 CNRS LETG/Rennes
}

\begin{abstract}
Résumé : Cet article présente une approche de modélisation multi-agents visant à simuler les relations entre climat local, cycle phénologique de la vigne et stratégies de productions viticoles. Cette démarche se place dans la perspective de mieux comprendre les ajustements des itinéraires agronomiques aux variations du climat. L'ensemble de la démarche est exposé dans le contexte d'une application en Argentine, dans la région de Mendoza.
\end{abstract}

\begin{abstract}
This paper presents a multi-agent approach to simulating the interactions between local climate, vine phenological cycle and wine production strategies. This approach is intended to provide a better understanding of agronomic itineraries adaptation to climate variations. This approach is presented in the context of an implementation in Argentina in the Mendoza area.
\end{abstract}

Mots clés : stratégies de production viticoles, changement climatique, modèle multi-agents, adaptation, dynamique de la vigne, scénarios

Keywords: wine-making production strategies, climate change, agent-based modeling, adaptation, wine-growing dynamics, scenarios

\section{INTRODUCTION}

Le changement climatique entraîne des répercussions sur les climats régionaux et locaux qui affectent les régions viticoles du monde entier (Jones, 2007 et 2015 ; Van Leeuwen et Darriet, 2016). Ces modifications à méso et micro-échelles soulèvent des enjeux à court terme concernant le maintien de la qualité et du style de vins mais également à plus long terme concernant l'adéquation des cépages et la durabilité économique des régions viticoles traditionnelles (Schultz et Jones 2010 ; Quénol et Bonnardot, 2014). Pour faire face à ces nouvelles contraintes environnementales et socio-économiques, les viticulteurs doivent reconsidérer leurs pratiques et leurs stratégies de production (Battaglini et al., 2009 ; Fraga et al., 2012 ; Ollat et al., 2016 ; Ashenfelter et Storchmann, 2016 ; Mosedale et al., 2016). La perspective du changement climatique est donc l'un des défis majeurs de l'industrie vitivinicole (Keller, 2010).

En Argentine, cinquième producteur de vins mondial (Canziani et Scarel, 2010), la problématique de l'adaptation de la viticulture entre en résonnance avec celle de l'accès à l'eau. Dans un pays où la quasi-totalité des vignobles sont irrigués (Ojeda et Saurin, 2014) et les besoins en eau des villes sont en croissance constante, la question de la gestion durable des ressources hydriques, qu'elles soient souterraines ou superficielles, est incontournable. 
Pour aborder les implications potentielles du changement climatique sur les activités viticoles, un modèle multi-agents a été développé dans le cadre du programme de recherche GICC TERADCLIM. Implémenté en partenariat avec la bodega Alta Vista, il aborde deux composantes essentielles de l'adaptation dans la région de Mendoza : l'évolution des conditions phénologiques (en relation avec les caractéristiques agroclimatiques des vignobles) et les stratégies agronomiques (qui se traduisent par une évolution des itinéraires techniques au regard des cépages implantés et de la variabilité du climat).

Cet article présente cette expérimentation dans le contexte spécifique de la région de Cuyo où la gestion du foncier viticole est en constante évolution, du fait des besoins d'irrigation et de la croissance urbaine.

\section{ConteXte de L'etude et de la viticulture en Argentine}

La viticulture s'est implantée en Amérique du Sud et notamment en Argentine lors des vagues de colonisation espagnole des XVème XVIème siècles. C'est au cours du XVIIème siècle que l'émergence de la région de Cuyo s'est opérée (Canziani et Scarel, 2010). Aujourd'hui avec près de 220000 ha cultivés, l'Argentine fait partie des 10 pays ayant la plus importante surface viticole $^{1}$ (Canziani et Scarel, 2010). La viticulture est présente dans 7 provinces qui comptent au moins 1000 ha en 2015 (figure 1) (Loussert, 2017). La principale province productrice de vins, Mendoza, présente un climat continental semi-aride caractérisé par des températures élevées en été et une pluviométrie faible d'environ $200 \mathrm{~mm}$ par an (Quénol et Bonnardot, 2014). La région étant majoritairement désertique, les exploitations viticoles se sont concentrées près des fleuves où l'irrigation s'est massivement développée. Cette configuration a permis la création d'oasis artificielles tournées vers la production de vins réputés (Vigil et al., 2003). Le cépage malbec est ainsi devenu un des marqueurs de l'identité viticole argentine (Griset et Laborie, 2016)

Pourtant, sur le plan agroclimatique, les conditions de cultures restent extrêmes. La région fait partie des zones chaudes à très chaudes selon les classifications des régions viticoles (Tonietto et Carbonneau, 2004 ; González et al., 2009). Associée à une diminution notable des précipitations hivernales dans les Andes projetées par les modèles climatiques (Chou et al., 2014, Barros et al., 2015), les capacités d'adaptation de la viticulture constituent une

\footnotetext{
${ }^{1}$ Liste des dix premiers pays viticoles du monde (surface exploitée en milliers d'hectares) : Espagne (969), Chine (875), France (789), Italie (702), Turquie (448), Etats-Unis (430), Argentine (219), Chili (212), Portugal (192) (OIV, 2019)
} 
problématique importante à l'échelle locale, régionale et nationale compte tenu du poids économique de cette activité.

La question de la gestion de l'eau, enjeu majeur dans l'oasis de Mendoza (Lavie, 2009), est ainsi à mettre en perspective avec les possibilités d'adaptation des systèmes de production actuels dans des contextes climatiques modifiés.

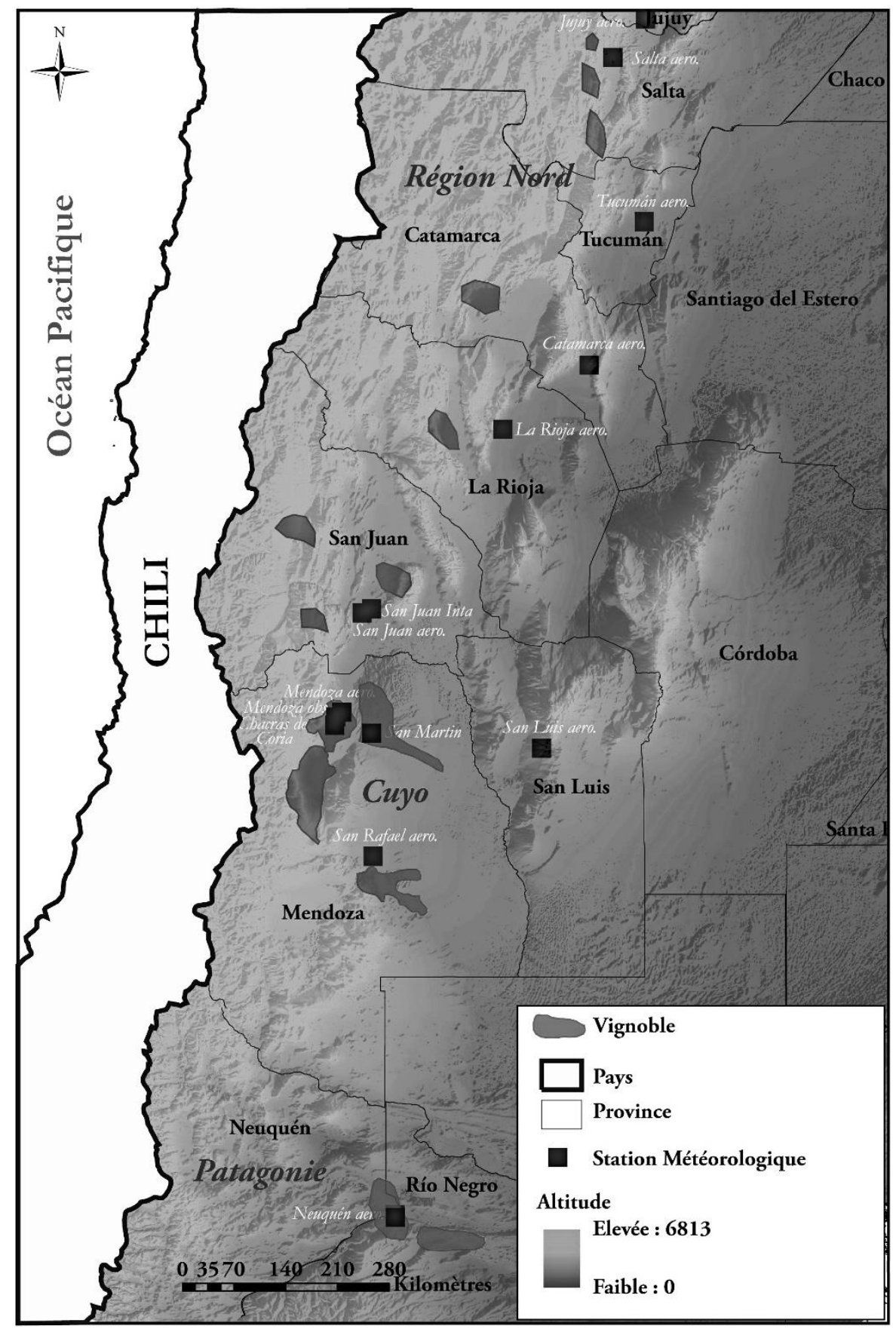

Figure 1 : Principales zones de production viticole en Argentine / Main wine-growing areas in Argentina

C'est dans ce contexte qu'une collaboration avec une bodega de la région de Mendoza s'est mise en place dans les années 2010. Elle visait à mieux caractériser le climat local et son effet 
sur la vigne en relation avec les caractéristiques des systèmes de production et des styles de vins produits. Pour répondre à cet objectif, un dispositif de mesures climatiques (15 data-logger enregistrant la température et 3 stations météorologiques complètes) et agronomiques a été mis en place dans les fincas (vignobles) de la bodega Alta Vista.

Le domaine de production s'étend sur 220 hectares localisés dans plusieurs secteurs viticoles de Mendoza (Lujan de Cuyo, Tupungato, la Vallée de Uco), de Salta et de Cafayate. Plusieurs sites pilotes ont été choisis et instrumentés dans la Province de Mendoza (figure 2).
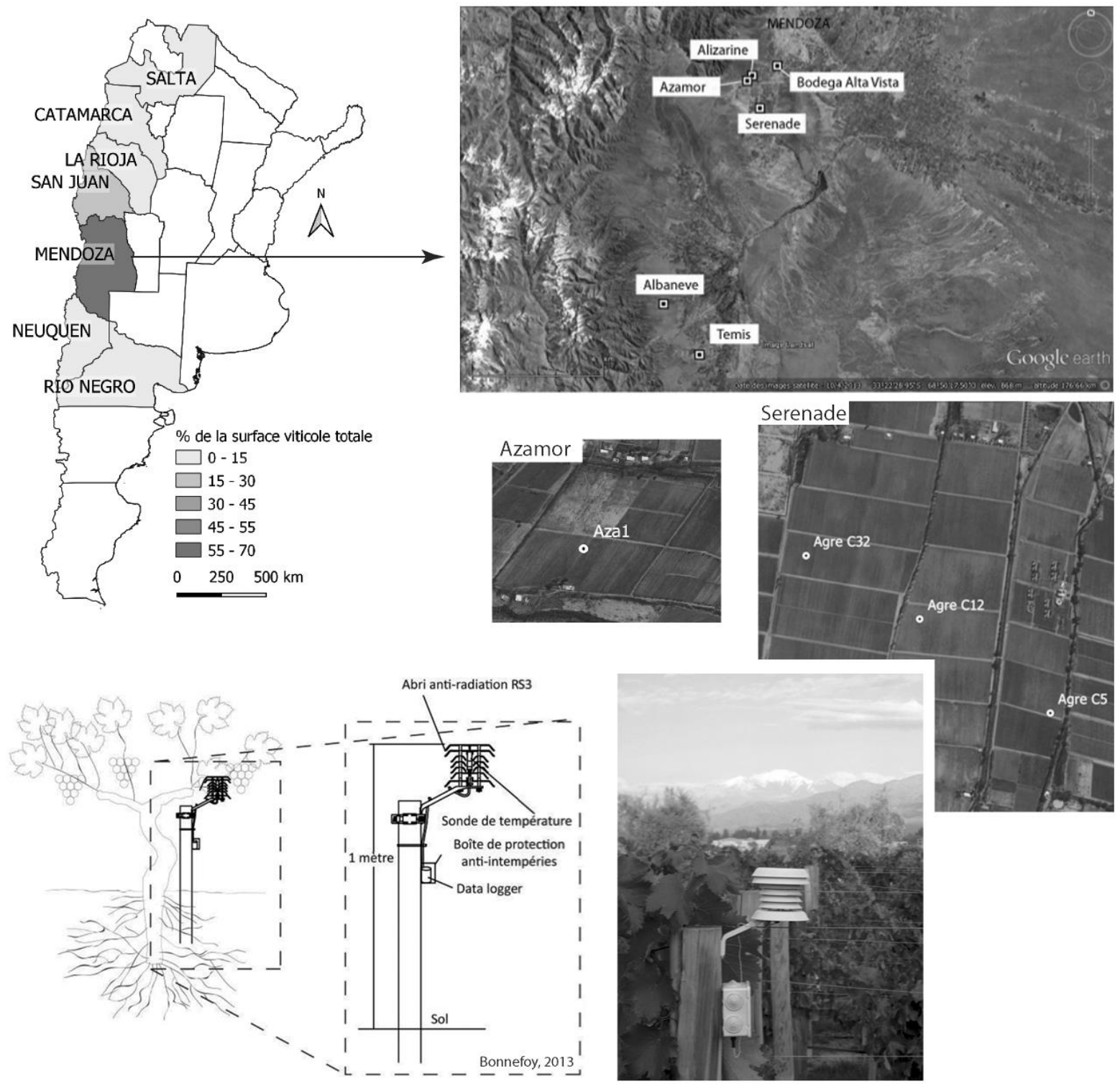

Figure 2 : Localisation des vignobles de la bodega Alta Vista et des postes de mesures / Location of the bodega Alta Vista vineyards and weather stations (data sources: Google Earth, Instituto Nacional de Vitivinicultura) 
Les capteurs de températures (de type Tiny Tag Gemini équipés d'un abri antiradiation RS3) ont été disposés en fonction des caractéristiques locales (topographie, exposition, type de sol...) susceptibles d'influencer les variables climatiques.

L'exploitation des données fournies par ce réseau de mesure montre une forte variabilité spatiale du climat à l'échelle locale. Ces résultats ont pu être mis en perspective avec les différences observées sur le terrain au niveau du cycle phénologique et des caractéristiques des baies de raisin (Quenol et al., 2014).

Cette première phase de recherche a été complétée par une approche de modélisation visant à simuler les relations entre climat local, cycle phénologique et stratégies de production. Cette démarche se place dans la perspective de mieux comprendre les ajustements des itinéraires agronomiques aux variations du climat sur la période historique, afin de projeter des trajectoires d'adaptation potentielles en fonction de différents scénarios du changement climatique.

\section{APPROCHE METHODOLOGIQUE}

Aborder dynamiquement l'évolution des conditions d'environnement et leurs implications sur la phénologie de la vigne et les caractéristiques des terroirs viticoles nécessitent de mettre en relation des informations hétérogènes sur le plan spatial et temporel. Elles concernent principalement des processus biophysiques, des observations agronomiques et des données climatiques acquises ou produites par modélisation à différentes échelles. La conduite d'une démarche de modélisation pose, dans ce contexte, des questions spécifiques relatives à la définition d'une granularité spatio-temporelle adaptée à la problématique viticole.

Pour présenter les choix de modélisation retenus dans cette expérimentation, l'utilisation du protocole Overview, Design concepts, Details (ODD, Grimm et al., 2006 et 2010) a été retenue. Les sections qui suivent correspondent aux différents éléments qui définissent ce protocole.

\subsection{Objectifs}

Dans cette recherche, la démarche proposée vise à lier les observations climatiques et agronomiques menées sur le terrain et les questionnements liés à la recherche d'une adaptation optimale de la vigne à la spécificité des terroirs viticoles locaux. Elle mobilise des technologies relevant de la géomatique et des modèles multi-agents.

L'objectif est double : 
- il s'agit d'une part de répondre aux questionnements des professionnels de la viticulture qui doivent adapter leurs méthodes de production à la variabilité des conditions climatiques, sans connaître l'horizon temporel de ces évolutions. Le développement de technologies permettant d'aborder dynamiquement et dans une dimension prospective cette problématique représente donc un fort enjeu à l'échelle locale (maintien de la spécificité des terroirs viticoles) et globale (identification de zones d'implantation en adéquation avec un cépage et des pratiques viticoles associées) ;

- il s'agit d'autre part de proposer une méthodologie de modélisation capable d'aborder une problématique complexe intégrant des contraintes spatio-temporelles multi-échelles permettant de restituer la croissance de la vigne à partir d'indices bioclimatiques et de modéliser les stratégies de production et d'adaptation dans différents contextes climatiques (approche rétrospective et prospective).

Pour répondre à ces deux objectifs, la stratégie a consisté à associer la bodega Alta Vista tout au long du projet depuis la fourniture de données, jusqu'à la formalisation des trajectoires agronomiques possibles pour différents scénarios de production. Si cette démarche présente certaines similitudes avec des expériences de co-construction du type COMMOD (Barreteau et al., 2010), elle ne peut être considérée comme une véritable approche de modélisation d'accompagnement, dans la mesure où seule une partie du processus de modélisation a été réalisée avec l'acteur (la conception et l'implémentation d'un modèle générique était déjà réalisé avant la prise de contact avec le viticulteur).

\subsection{Entités, variables d'états et échelles}

Le modèle baptisé DAHU-Vigne-AV a été implémenté dans l'environnement NetLogo ${ }^{2}$. Il est composé de trois classes d'agents génériques issus de la plateforme DAHU (Dynamique des Activités Humaines, Tissot et al., 2005, Tissot, 2018) : Superviseurs, Exploitants et Production. Les Agents "Superviseurs" représentent les structures de régulation. Ils orientent et contrôlent le bon déroulement des activités. Les Agents "Exploitants" restituent le déroulement des pratiques associées à chaque activité. Ils possèdent des objectifs et élaborent une stratégie. Enfin, les Agents "Production" ont pour objectif de produire des ressources dans un environnement contraint. Ils représentent l'élément central du simulateur car ils déterminent la

\footnotetext{
${ }^{2}$ NetLogo est un environnement de modélisation programmable libre permettant de simuler des phénomènes naturels et sociaux (Sklar, 2011). Il a été créé par Uri Wilenski en 1999 et son développement est poursuivi de manière continue par le Center for Connected Learning and Computer-Based Modeling.
} 
nature et la qualité des ressources disponibles pour les Agents "Exploitants". Ils jouent donc un rôle essentiel dans la stratégie des Agents "Exploitants" et dans l'attitude des Agents "Superviseurs" qui, en fonction de l'état de cette ressource ou de son environnement, peuvent prendre des mesures d'adaptation. Ces capacités réactives se manifestent par un comportement adaptatif en fonction de l'évolution des contraintes d'environnement qui, dans le cas présent, sont fournies par une base de données spatio-temporelle couplée au modèle.

Ces trois classes génériques ont été transcrites pour répondre aux besoins spécifiques de la modélisation des activités viticoles. Trois classes principales d'agents ont ainsi été implémentées (figure 3) :

- l'Agent "Regulateur" a un rôle de superviseur au sein du modèle. Il fixe notamment les quotas d'eau attribués pour l'irrigation. Il est en relation directe avec les Agents "Vignes" qui lui transmettent des informations relatives à leur exposition au stress hydrique. En fonction de ces informations, l'agent "Regulateur" peut modifier les droits d'accès à l'eau ;

- les Agents "Viticulteurs" ont pour objectif de produire un raisin conforme à un cahier des charges spécifique en fonction du style de vin souhaité. Cette action implique de cultiver la vigne dans des conditions optimales, compte tenu des spécificités agronomiques des parcelles, du mode de production et de la stratégie du viticulteur ;

- les Agents "Vignes" sont des entités de production du raisin. Elles correspondent à des entités spatiales de type parcelle pouvant appartenir ou non à une zone d'appellation. Ces agents ont pour rôle de restituer la dynamique de croissance de la vigne en fonction des conditions climatiques.

D’autres classes d'agents viennent enrichir cette base générique :

- les agents "Viticulteurs" disposent d'agents "Ouvriers viticoles" et "Tracteurs" qui leurs permettent de mettre en œuvre les actions agronomiques ;

- les agents "Vignes" sont associées à un agent "Pathogène" qui restitue la dynamique de certaines maladies comme le mildiou en fonction des conditions climatiques ;

- enfin, des agents "Capteurs" fournissent des informations climatiques (données météorologiques, indices bioclimatiques) utiles aux agents "Vignes", "Viticulteurs" et "Regulateur". 
L'environnement dans lequel sont situés les agents est composé d'entités spatiales surfaciques (parcelles et zones de production de la Bodega Alta Vista) et ponctuelles (capteurs, ouvriers, tracteurs).

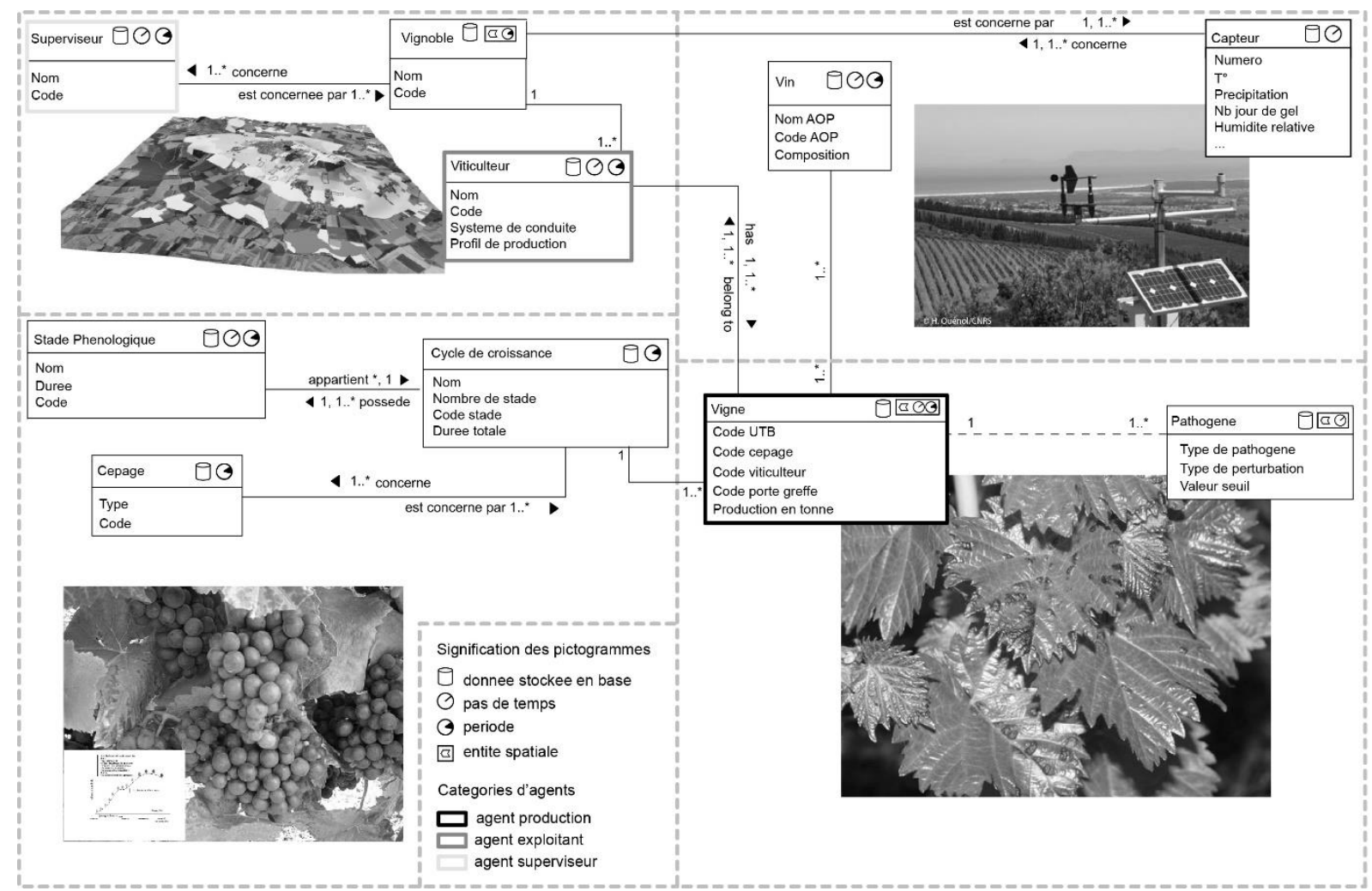

Figure 3 : Architecture du modèle DAHU-Vigne-AV / Framework of DAHU-Vigne-AV model

Les attributs et paramètres du modèle ont été spécifiés à partir d'une base de connaissance (données des enquêtes, du milieu naturel, etc.) regroupant l'ensemble des informations permettant de décrire les pratiques et d'expliquer la relation entre la vigne et le climat. L'objectif était de produire une description fine du déroulement des activités viticoles en les replaçant dans un contexte de changement climatique.

Pour cela, deux enquêtes approfondies (tableau 1) ont été réalisées auprès des responsables de la production viticole de la bodega Alta Vista afin de bien comprendre les interactions entre la variabilité climatique et les pratiques culturales (annuelles et pérennes).

La première étape consistait à évaluer l'influence des conditions météorologiques sur les pratiques annuelles. Cette enquête semi-dirigée traitait les questions et les thèmes concernant les périodes favorables et défavorables de travail, les techniques et les machines impliquées et l'influence des conditions climatiques sur la période de travail. La seconde étape visait à évaluer les capacités d'adaptation du viticulteur. Des questions sur les changements de pratiques et les facteurs menant à ces changements ont été plus spécifiquement ciblées. En complément, les 
conditions climatiques qui caractérisent les «bons» et les «mauvais » millésimes et les stratégies d'adaptation adoptées au cours de ces années de références ont été évoquées.

\begin{tabular}{|c|c|c|}
\hline & Enquête 1 & Enquête 2 \\
\hline Objectifs & $\begin{array}{l}\text { Etude de la sensibilité des } \\
\text { pratiques annuelles aux } \\
\text { conditions climatiques }\end{array}$ & $\begin{array}{l}\text { Etude de la capacité } \\
\text { d'adaptation des viticulteurs }\end{array}$ \\
\hline Méthode & Semi-dirigée & Semi-dirigée \\
\hline $\begin{array}{l}\text { Questions et } \\
\text { thèmes } \\
\text { abordées }\end{array}$ & $\begin{array}{l}\text { - Périodes de travail } \\
\text { - Techniques et machines } \\
\text { impliquées } \\
\text { - Variables climatiques } \\
\text { favorables et défavorables }\end{array}$ & $\begin{array}{l}\text { - Comment les pratiques ont évolué } \\
\text { - Facteurs menant aux changements } \\
\text { - Conditions climatiques caractérisant } \\
\text { «bon » et «mauvais » millésimes } \\
\text { - Stratégies d'adaptation }\end{array}$ \\
\hline
\end{tabular}

Tableau 1 : Caractéristiques des enquêtes réalisées auprès du viticulteur / Characteristics of the surveys carried out with the winegrower.

En complément, l'ensemble des données biophysiques et les paramètres agronomiques locaux de la vigne nous ont ainsi été fournis par la bodega. Ces informations ont permis de reconstituer le cycle végétatif de la vigne et d'associer des actions de conduites agronomiques à des paramètres d'environnement contrôlés par l'agent "Viticulteurs".

Tous ces éléments ont été recueillis au sein d'une base de données au format postgesq1/postgis qui permet d'administrer les informations collectées et d'y associer des couches d'information géographique. Cette configuration offre la possibilité de lier des variables de terrain à des objets spatiaux et de réaliser des requêtes thématiques pour initialiser et paramétrer le modèle. Le couplage entre modèle et base de données s'effectue via l'utilisation des extensions "gis" et "sql" de Netlogo.

La séquence de modélisation combine ainsi des informations géographiques numériques classiques (modèle numérique de terrain, images satellitaires, orthophotographies, données d'observations agronomiques et climatiques géoréférencées) avec des données à dire d'acteurs (itinéraires agronomiques, perceptions du changement climatique, stratégies de production).

\subsection{Processus et ordonnancement du modèle}

Les agents sont ordonnancés au pas de temps journalier. A chaque pas de temps, les processus sont réalisés dans l'ordre suivant :

- les agents "Capteurs" mettent à jour l'ensemble des données météorologiques et calculent les indices bioclimatiques ; 
- les agents "Vignes" simulent le cycle phénologique et actualisent le stade de croissance de la plante ;

- l'agent "Pathogène" reste en dormance ou augmente son énergie en fonction des conditions climatiques ;

- les valeurs de balance hydriques sont contrôlées par les agents "Vignes" et "Viticulteurs"

- le statut prioritaire des agents "Vignes" est mis à jour ;

- les actions agronomiques sont choisies en fonction du stade de croissance de la vigne ;

- les actions agronomiques sont exécutées ou misent en attente ;

- les données agronomiques (stress hydriques, actions réalisées, en cours ou en attente) sont mises à jour dans la base postgresq1/postgis.

\subsection{Principaux éléments de conception}

Le modèle est basé sur une recherche systématique de l'optimisation de l'itinéraire agronomique en fonction de l'évolution des conditions climatiques. Aucune alternative liée à une délocalisation spatiale de sites de production n'est proposée. Ce choix peut paraitre restrictif mais il répond au besoin d'analyser les principaux leviers et mécanismes d'adaptation des systèmes de production actuels. Si l'on se réfère à la nomenclature proposée par Edmond (Edmond et al., 2019), le prototype DAHU-Vigne-AV peut ainsi être considéré comme un modèle explicatif, visant à mieux comprendre les processus et variables à l'origine des changements opérés dans les choix de conduites agronomiques.

\subsection{Emergence}

Il n'existe pas de dynamique spatiale dans ce modèle dans la mesure où les zones de production sont fixes. En revanche, les simulations mettent en évidences des changements de comportements basés sur des anticipations, en fonction des contraintes climatiques influençant la stratégie de production de l'agent "Viticulteurs".

\subsection{Perception}

Les agents "Vignes", "Viticulteurs", et "Superviseurs" ont la capacité de percevoir l'évolution des conditions climatiques en contrôlant des indicateurs à différents niveaux d'échelles (échelle régionale pour "Superviseurs", échelle locale pour "Vignes" et "Viticulteurs"). Les agents 
"Viticulteurs" perçoivent le cycle phénologique et l'état hydrique de leurs parcelles. Les agents "Vignes" perçoivent le risque pathogène.

\subsection{Interactions}

Les interactions interviennent principalement dans les méthodes d'exécution des actions agronomiques. Elles concernent en premier lieu les agents "Vignes" et "Pathogène". En condition climatique favorable, le pathogène sort de dormance et monte en énergie jusqu'à un seuil considéré comme critique. Cette situation entraîne une demande de traitement phytosanitaire (par l'agent "Vignes" concerné) à l'agent "Viticulteurs". Si l'action est réalisée, le pathogène retourne en dormance pendant une période donnée (dépendant du type de produit utilisé). D’autres interactions sont présentes entre les agents "Vignes" et "Viticulteurs" concernant l'exécution des actions agronomiques. Les parcelles viticoles sont classées prioritairement en fonction de l'avancement de leur cycle phénologique. Les agents "Vignes" effectuent des demandes d'ouvriers et/ou de tracteurs en fonction des actions à réaliser. En retour, le viticulteur alloue ou non des moyens à l'exécution de l'action en fonction de sa stratégie individuelle. Le statut prioritaire de la parcelle évolue en fonction de cette interaction.

\subsection{Stochasticité}

Le modèle intègre des processus stochastiques dans la dynamique de certains agents. Il s'agit notamment des agents "Pathogène", dont la sortie de dormance et la montée en énergie peuvent être aléatoires, et des agents "Viticulteurs" qui peuvent opérer des choix probabilistes dans l'exécution des actions agronomiques non indispensables à la conduite de la vigne.

\subsection{Observation}

L'ensemble des paramètres et des actions réalisées par les agents peuvent être contrôlés via l'interface graphique du modèle. La plateforme NetLogo, utilisée comme support de développement, facilite la visualisation des résultats en cours de simulation via un affichage cartographique mis à jour à chaque pas de temps (figure 4). Le déroulement des actions agronomiques peut ainsi être suivi en temps réel. Des sondes permettent également de visualiser sous une forme graphique de nombreux paramètres comme des indices bioclimatiques, l'état de la balance hydrique, le nombre d'actions agronomiques... 


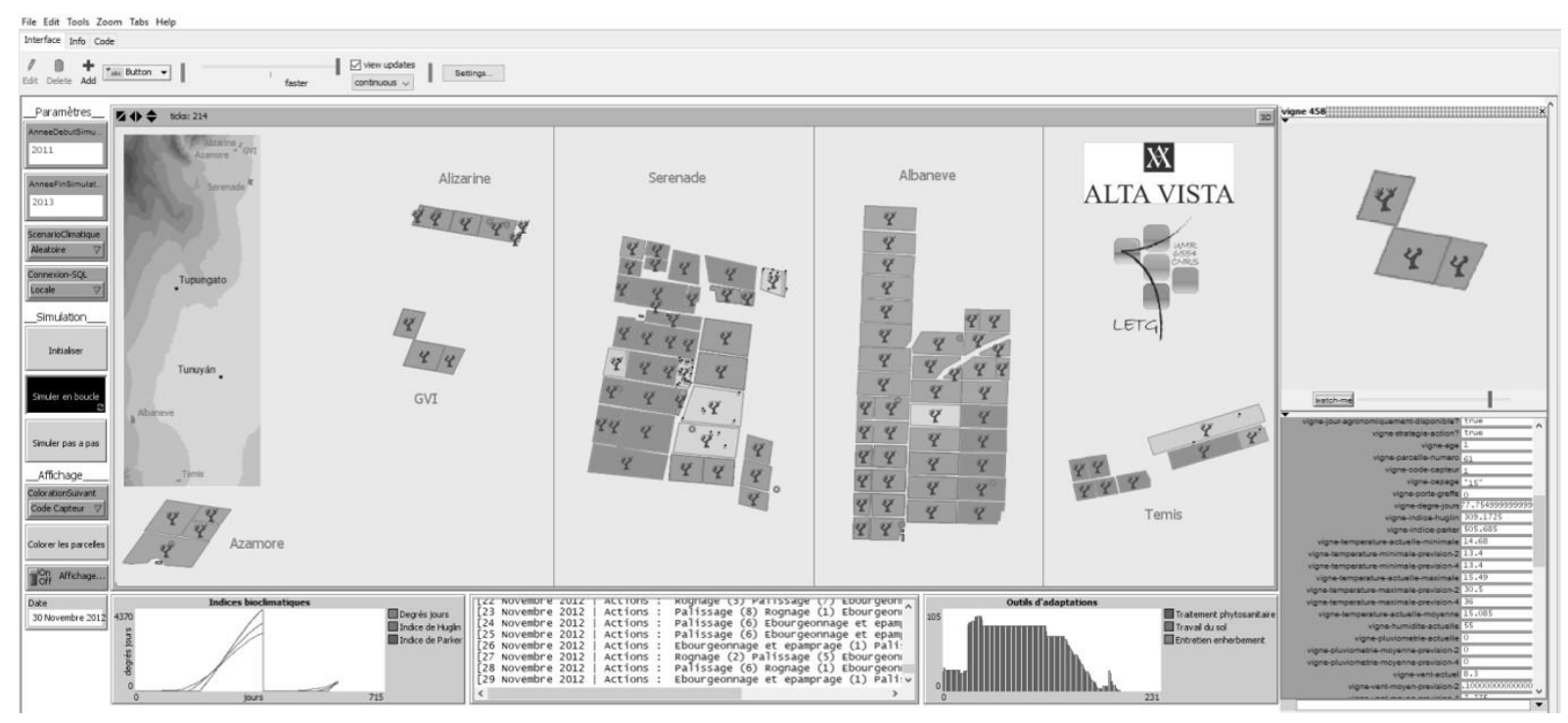

Figure 4 : Capture d'écran du modèle DAHU-Vigne-AV / Screenshot of DAHU-Vigne-AV model

Ces outils sont une aide au contrôle du bon déroulement de la simulation et un support de communication vers la profession viticole. Ils restent néanmoins perfectibles car ils proposent une vision instantanée des sorties de calcul du modèle et nécessitent d'être complétés par de nombreuses analyses a postériori pour expliciter les résultats obtenus.

\section{DETAILS DE LA SEQUENCE DE SIMUlation}

L'objectif des simulations est de mettre en relation le cycle phénologique de la vigne avec les conditions climatiques et les modes de conduites agronomiques. Un schéma de synthèse permet de résumer l'ensemble de cette séquence (figure 5).

\subsection{Initialisation}

La procédure de simulation débute par une phase d'initialisation qui créé l'ensemble des agents manipulés par le modèle. Elle donne lieu au chargement des objets spatiaux (zone de productions, bâtiments, parcelles, capteurs) dans l'interface graphique de Netlogo. Des données d'initialisation sont fournies aux différents agents à partir de listes de données construites par des requêtes sql. 


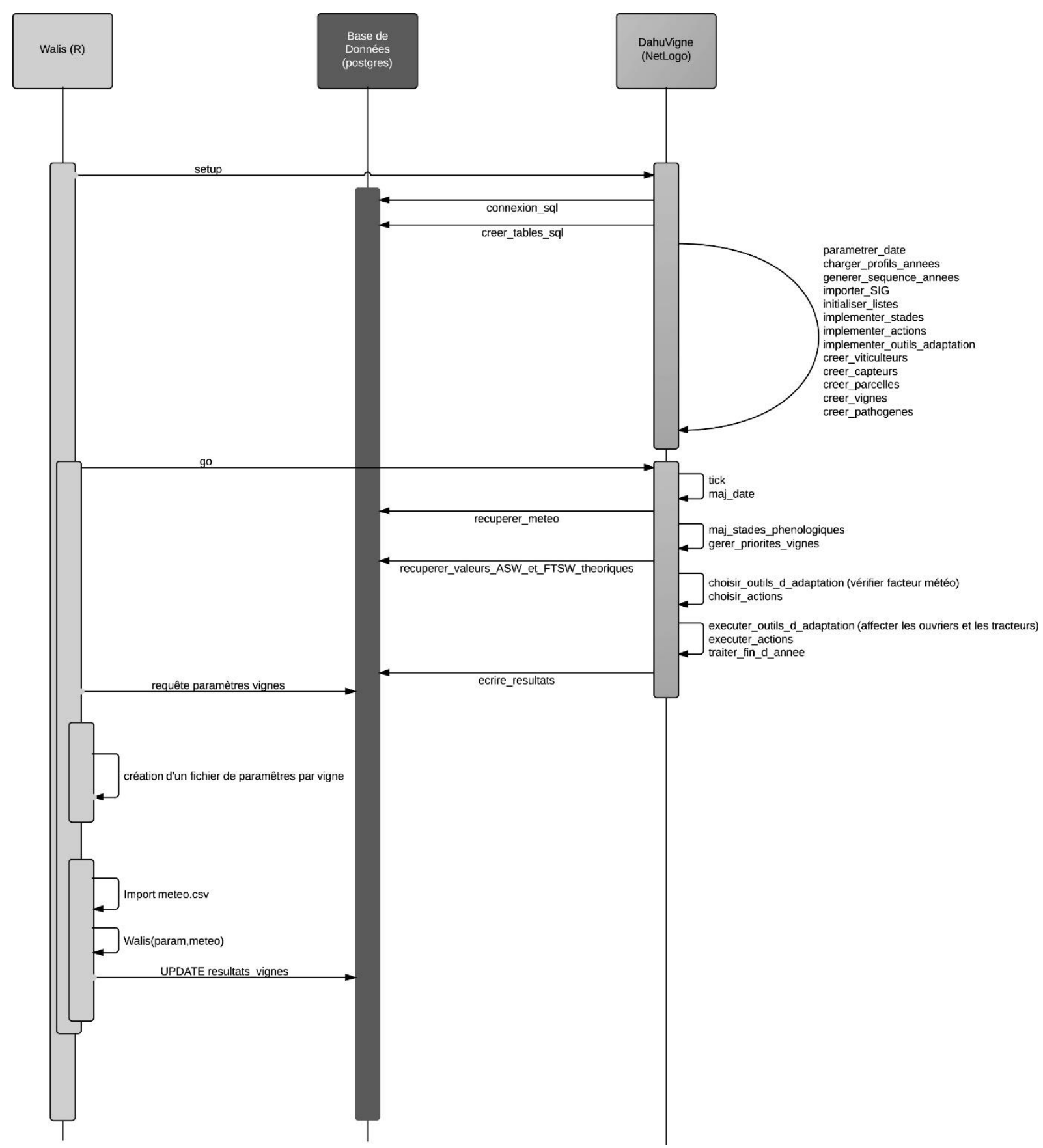

Figure 5 : Diagramme de séquence de la simulation / simulation sequence diagram

\subsection{Données d'entrée}

Les données de calibration et de forçage des agents sont intégrées dans le modèle via des requêtes sql dans la base de connaissance.

L'agent "Regulateur" est initialisé avec des objectifs de contrôle de la ressource en eau.

Les agents "Vignes" sont affectés de variables agro-environnementales concernant le type de cépage, le type de sol et ses caractéristiques physiques (indice de pente, réserve en eau) ainsi que les données météorologiques de la période considérée. 
Les agents "Viticulteurs" sont créés à partir des données d'enquêtes qui permettent de calculer des calendriers de pratique et de construire des arbres de décisions sous contraintes agroclimatiques en fonction de stratégies individuelles.

Les données climatiques sont intégrées en fonction du choix de réaliser une simulation rétrospective ou prospective. Dans le premier cas, le modèle intègre des données historiques provenant de stations météorologiques de références et des capteurs Tiny Tag (lorsque les données sont disponibles). Elles fournissent des valeurs de températures, précipitations, vitesse du vent, humidité et évapotranspiration potentielle. Ces données sont affectées à une parcelle en fonction de son implantation géographique (distance par rapport aux stations et capteurs). Elles permettent de calculer l'ensemble des indices bioclimatiques utiles au modèle pour simuler la croissance de la vigne et donnent des informations indispensables à l'agent "Viticulteurs" pour conduire des actions agronomiques et déterminer sa stratégie.

Dans le second cas, l'utilisation des sorties proposées par les modèles climatiques régionalisés reste peu pertinente en raison de l'imprécision de certaines données simulées comme l'ETP où les précipitations. Pour contourner cette limite, une méthode alternative a été mise en œuvre. Après avoir réalisé une classification des vingt dernières années en fonction de leurs profils climatiques (calcul des variables du climat et des indices bioclimatiques propres à la vigne), un ensemble de scénarios hypothétiques ont été construits. Ils intègrent différentes tendances de changement et peuvent être introduits dans le modèle de manière aléatoire ou choisie par l'utilisateur. Le choix d'un scénario chaud et sec, par exemple, se traduira au niveau de la modélisation par l'intégration majoritaire de données issues d'années chaudes et sèches (classées en fonction de la typologie) au cours de la période de simulation. Cette procédure totalement probabiliste peut être paramétrée par l'utilisateur en fonction du type de scénario privilégié.

\subsection{Sous-modèles}

Le prototype DAHU-Vigne-AV comporte trois sous modèles.

\subsubsection{Phénologie}

La dynamique de croissance de la vigne fait appel à des algorithmes simples permettant de restituer le cycle végétatif à partir d'indices bioclimatiques. De nombreuses études ont montré que l'accumulation de chaleur est significativement corrélée aux principaux stades phénologiques de la vigne, comme la floraison ou la véraison (Jones et Davis, 2000 ; Van Leeuwen et al., 2008 ; Duchêne et Schneider, 2005 ; Parker et al., 2013). Le découpage en 
stades phénologiques suivant la classification de Baggiolini (Baggiolini, 1952) a été utilisé pour matérialiser cette croissance (figure 6).

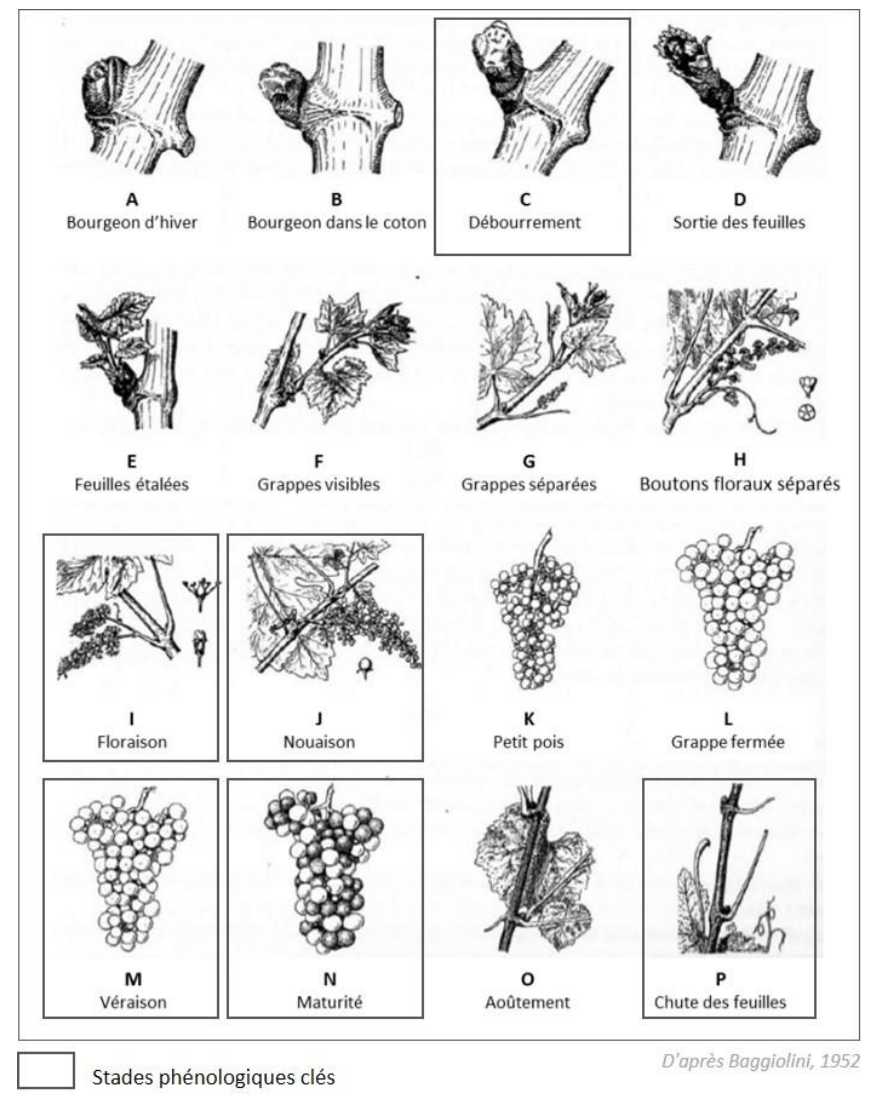

\begin{tabular}{|c|c|c|c|}
\hline Cépage & Stade & GDD* & GFV** \\
\hline \multirow{16}{*}{ Malbec } & $A$ & 0 & - \\
\hline & $B$ & 25 & - \\
\hline & C & 50 & - \\
\hline & $D$ & 102 & - \\
\hline & $E$ & 154 & - \\
\hline & $F$ & 206 & - \\
\hline & $G$ & 258 & - \\
\hline & $H$ & 310 & - \\
\hline & 1 & 362 & 1306 \\
\hline & $J$ & 532 & 1830 \\
\hline & $K$ & 703 & 2353 \\
\hline & $L$ & 873 & 2877 \\
\hline & $M$ & 1043 & 3400 \\
\hline & $N$ & 1470 & - \\
\hline & 0 & - & - \\
\hline & $P$ & - & \\
\hline
\end{tabular}

Figure 6: Classification de Baggiolini et relations entre stades et indices bioclimatiques en fonction d'un cépage. Exemple pour le cépage Malbec, emblématique de cette zone de production (* Growing Degree Day, ** Grapevine Floraison Veraison Model) / Baggiolini classification and relationships between stages and bioclimatic indices according to a grape variety. Example for the Malbec, emblematic grape variety of this production area (* Growing Degree Day, ** Grapevine Floraison Veraison Model)

Le passage d'un stade à l'autre est calculé à partir de l'indice de degré-jours (GDD), tel que :

Indice de degré-jours $(\mathrm{GDD})=$ Somme de la température moyenne $[(\mathrm{Tx}+\mathrm{Tn}) / 2]-10^{\circ} \mathrm{C}$ (température de base) calculée du 1er septembre au 31 mars pour 1'hémisphère sud (Van Leeuwen et al., 2004).

La valeur de cet indice est calculée quotidiennement par les agents "Vignes" afin de savoir si la vigne est apte, ou non, à passer au stade suivant. Les données de température sont fournies par les 15 capteurs et les trois stations météorologiques qui reflètent les spécificités et la variabilité locale de la température dans les vignobles d'Alta Vista en fonction de la topographie, de l'exposition à l'ensoleillement, de la morphologie de la végétation, etc. 
Concernant les stades de floraison et véraison, et compte tenu de sa bonne corrélation avec les cépages argentins, le modèle Grapevine Floraison Veraison (GFV) (Parker et al., 2013) a été utilisé. Il correspond à un cumul des températures moyennes en base 0 (du $1^{\mathrm{er}}$ septembre au 31 mars pour 1'hémisphère sud).

Les données de température permettent également de calculer l'indice héliothermique de Huglin (Huglin, 1998), qui est utilisé par le modèle pour déterminer la date de vendange potentielle (le lancement de la vendange dépend de la prise de décision du viticulteur). La valeur de l'Indice de Huglin pour une station correspond à la valeur cumulée de l'indice à la date du 31 mars, le cumul se faisant depuis le $1^{\mathrm{er}}$ octobre (pour l'hémisphère Nord, l'indice est calculé sur la période du $1^{\mathrm{er}}$ avril au 30 septembre) à l'aide de la formule suivante :

$\mathrm{IH}=\Sigma[(\mathrm{Tm}-10)+(\mathrm{Tx}-10) / 2] * \mathrm{k}$

où $\mathrm{Tm}=$ Température moyenne, $\mathrm{Tx}=$ Température maximale et $\mathrm{k}$ le coefficient de longueur $\mathrm{du}$ jour variant de 1,02 à 1,06 (en fonction de la latitude de la zone d'intérêt).

\subsubsection{Bilan hydrique}

Pour caractériser l'impact du climat local sur la dynamique de la vigne, le modèle SEVE a été couplé au modèle de bilan hydrique WAter Balance for Intercropping Systems (WALIS) (Celette et al., 2010). Développé en langage R, ce modèle permet de simuler la disponibilité en eau du sol à un pas de temps quotidien. Implémenté à l'échelle de la parcelle, WALIS permet de simuler l'évolution quotidienne de la teneur en eau du sol en fonction des besoins en eau de la vigne et des cultures enherbées en concurrence le cas échéant. L'eau du sol est représentée par un réservoir caractérisé par son eau transpirable totale (TTSW), représentant la différence entre la teneur en eau maximale et minimale (extractible), l'eau transpirable du sol (TSW) et la fraction d'eau transpirable du sol (FTSW = TSW/TTSW). Le FTSW est donc un indicateur de l'état hydrique de la vigne pour gérer le système d'irrigation de la vigne dans le modèle DAHUVigne-AV. Le couplage des deux modèles est réalisé via l'utilisation de l'extension RNetLogo, qui fournit au logiciel $\mathrm{R}$ une interface vers NetLogo, et le partage de la base de données postgresql/postgis qui permet l'échange des données d'initialisation et de forçage. Les sorties du modèle WALIS permettent d'informer les agents "Vignes" de leur état de stress hydrique. Cette variable est utilisée pour le lancement d'actions spécifiques, par exemple un travail superficiel du sol en cas de stress hydrique modéré ou le déclenchement de l'irrigation en situation de stress critique. 


\subsubsection{Stratégies de production et prise de décision}

Le processus de prise de décision est segmenté en différentes phases permettant d'établir des priorités d'actions agronomiques en fonction des caractéristiques de chaque parcelle (phénologie, niveau de stress hydrique, sensibilité aux pathogènes), des contraintes techniques propres à chaque action (disponibilité de la main d'œuvre ou du matériel nécessaire) et des conditions météorologiques (favorables ou défavorables à l'exécution de l'action) (figure 7).

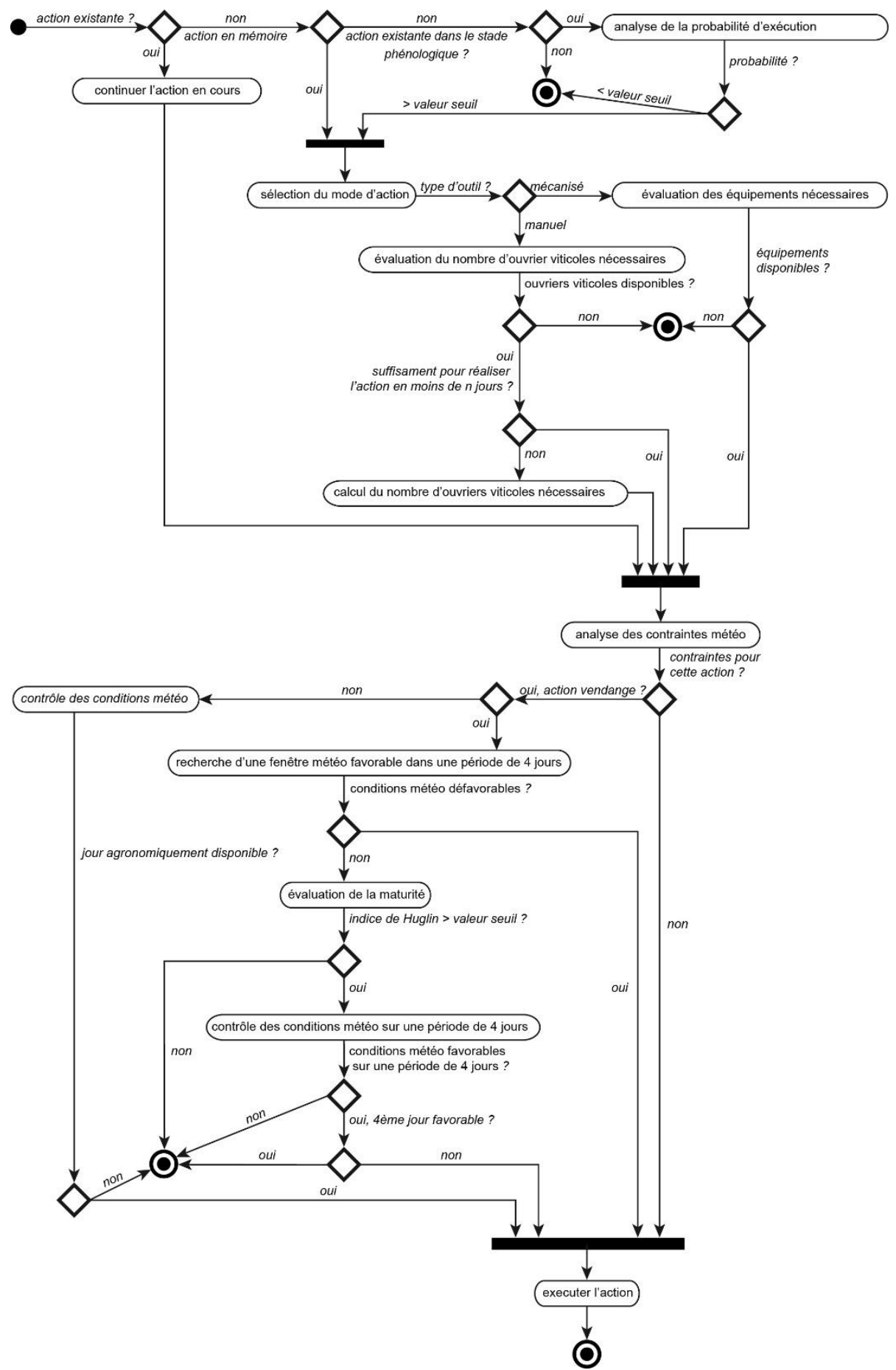

Figure 7: Processus de prise de décision d'un viticulteur (Tissot et al., 2017) / winegrower's decision-making process 
Les données de températures et de précipitations sont utilisées pour calculer des jours "agronomiquement disponibles" (Barbeau et al., 2014) pour la réalisation des tâches de travail de la vigne. En fonction de ces données, de ses objectifs de production et des caractéristiques agroclimatiques de ses parcelles (nature du sol, pente, exposition, bilan hydrique...), chaque Agent "Viticulteurs" réalise des choix de conduite agronomique (figure 8). La variabilité des conditions météorologiques agit donc directement et en temps réel (du modèle) sur la dynamique de la vigne et le comportement de l'agent "Viticulteurs". Dans ce contexte, une fenêtre de connaissance des données à $\mathrm{J}+4$ est fournie aux agents "Viticulteurs" de manière à leur donner les moyens d'établir une stratégie prévisionniste.

Parmi les actions que les viticulteurs vont pouvoir mener, certaines vont jouer directement un rôle sur la conduite de la vigne, d'autres vont faciliter son développement (pratique de l'enherbement concurrent, travail du sol, irrigation). L'ensemble de ces éléments détermine des itinéraires de conduites qui sont ajustés en temps réel dans le modèle. Autrement dit, les actions réalisées à un pas de temps t peuvent avoir une influence sur les choix opérés à $t+n$.

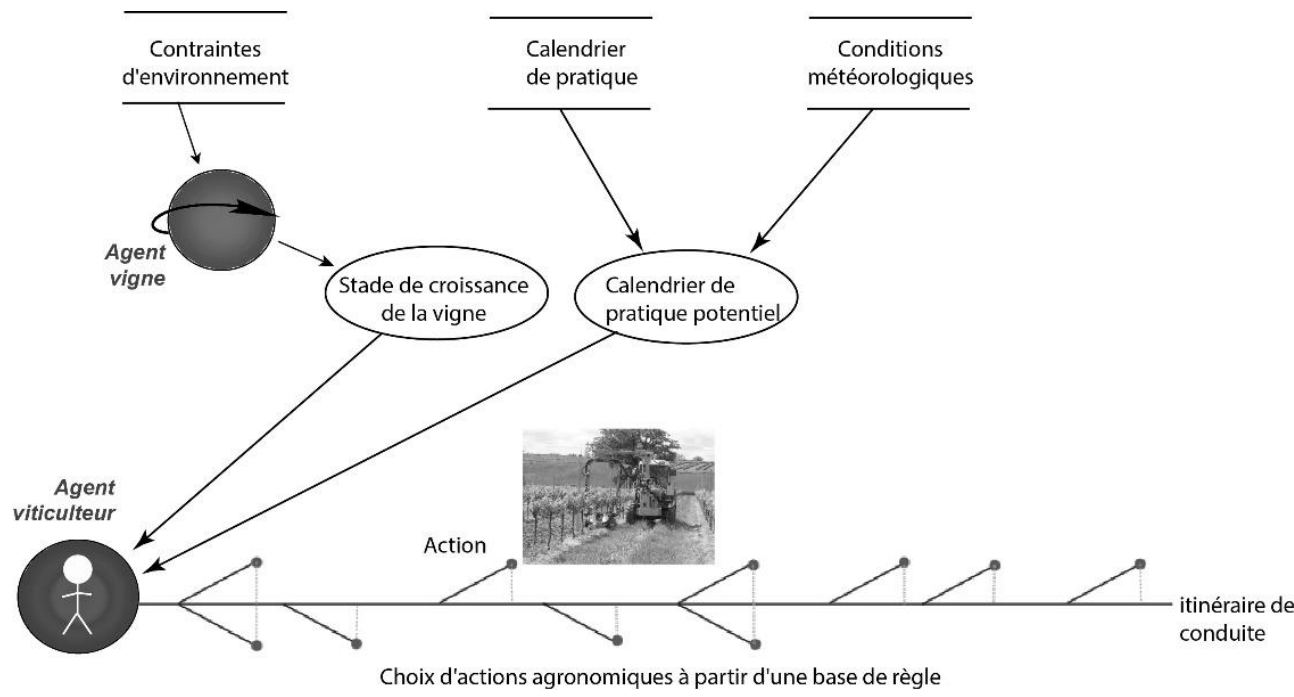

Figure 8: Contraintes et processus alimentant la prise de décision de de l'agent "Viticulteurs" / Constraints and processes leeding to winegrowers decision making

Cette étape de modélisation, nécessite d'être validée en raison de la complexité de la chaîne de relations aboutissant à la prise de décision. Dans ce contexte, des interactions régulières avec la bodega Alta Vista ont permis d'ajuster les mécanismes orientant leurs choix d'actions agronomiques. Ainsi, les choix réalisés peuvent être différents selon la zone de production concernée, certains sites étant clairement privilégiés en raison de leur aptitude à produire un raisin de grande qualité. Certaines actions peuvent donc être prioritaires sur les parcelles concernées. 


\section{Principaux Resultats}

Le modèle DAHU-Vigne-AV fournit trois types de résultats. Il restitue tout d'abord les différentes phases du cycle phénologique en fournissant des dates de changements de stade selon la classification de Baggiolini. Ces résultats ont été comparés aux observations phénologiques menées sur le terrain par les agronomes de la bodega Alta Vista. Les données obtenues confortent la bonne corrélation des indices bioclimatiques avec le cycle de croissance de la plante puisque les écarts sont inférieurs à 6 jours, quelle que soit la parcelle concernée.

Le deuxième type de résultat concerne l'itinéraire technique mené au cours de l'année (figure 9). Les données sont présentées sous la forme d'une séquence de pratiques qui comprend des actions classiques de conduite de la vigne et des outils d'adaptation comme l'irrigation ou différents modes de gestion des inter-rangs (plantation d'un enherbement concurrent, travail du sol).

Pour parvenir à simuler ces actions, plusieurs phases de calibration ont été nécessaires afin de déterminer les valeurs seuils menant au choix d'une action et les contraintes qui en contrôlent l'exécution. Plutôt que de conduire des tests de sensibilité pour chacune des actions (qui auraient entraîné des temps de calcul considérables), nous avons sollicité les viticulteurs de la bodega Alta Vista pour définir puis affiner ces paramètres. Au-delà de l'étape de calibration, cette connaissance experte a été un atout indéniable pour identifier les limites du modèle dans sa capacité à reconstituer une prise de décision fondée sur des paramètres environnementaux (cycle phénologique, indices bioclimatiques, données météorologiques...).

Les résultats obtenus sur les années 2013 et 2014 pour lesquelles nous disposions de données de terrain précises sur le suivi des actions (calendrier de pratiques et observations phénologiques) montrent une cohérence variable des résultats.

Si la séquence d'action est bien simulée, les dates de certaines pratiques sont mal estimées par le modèle car elle relève d'une prise de décision complexe, difficile à paramétrer. C'est notamment le cas de la taille qui peut intervenir sur une longue période (durant toute la phase de bourgeon d'hiver) et être retardée ou anticipée selon la stratégie du viticulteur (favoriser ou non une maturité précoce ou tardive) et le style de vin qui est ciblé. Des actions comme l'effeuillage sont également complexes à restituer car elles dépendent à la fois du niveau des températures (contrôle de l'exposition des baies aux très fortes chaleurs) et de l'état de stress hydrique de la plante. 


\begin{tabular}{|c|c|c|}
\hline code_parcelle & action & \\
\hline 97 & 17/05/2013 Taille & \\
\hline 97 & $12 / 08 / 2013$ Fertilisation du sol & \\
\hline 97 & 09/09/2013 Desherbage du cavaillon & \\
\hline 97 & 11/10/2013 Ebourgeonnage et epamprage & \\
\hline 97 & 07/11/2013 Rognage & Liste des actions proposées \\
\hline 97 & 09/11/2013 Effeuillage & Pretaille \\
\hline 97 & 12/11/2013 Palissage & Taille \\
\hline 97 & 20/11/2013 Traitement phytosanitaire & Broyage des sarments \\
\hline 97 & 04/12/2013 Rognage & Fertilisation du sol \\
\hline 97 & $17 / 12 / 2013$ Irrigation & Desherbage du cavaillon \\
\hline 97 & 24/12/2013 Traitement phytosanitaire & Ebourgeonnage et epamprage \\
\hline 97 & 02/01/2014 Irrigation & Palissage \\
\hline 97 & 05/02/2014 Irrigation & Rognage \\
\hline 97 & 04/03/2014 Vendange & Effeuillage \\
\hline 97 & 28/04/2014 Irrigation & Eclaircissage \\
\hline 97 & 20/05/2014 Irrigation & Vendange \\
\hline 97 & 26/08/2014 Irrigation & Traitement phytosanitaire \\
\hline 97 & $\begin{array}{l}16 / 09 / 2014 \text { Irrigation } \\
11 / 10 / 2014 \text { Irrigation }\end{array}$ & Travail du sol \\
\hline 97 & $21 / 11 / 2014$ Irrigation & Entretien d'un enherbement conccurent \\
\hline 97 & $17 / 12 / 2014$ Irrigation & Irrigation \\
\hline
\end{tabular}

Figure 9 : Exemple d'un itinéraire technique simulé par le modèle / Example of a technical itinerary simulated by the model

L'absence d'action de travail du sol (qui permet de diminuer le stress hydrique modéré) s'explique par le contrôle systématique de la balance hydrique par l'irrigation. Dans ce contexte, le modèle déclenche un apport d'eau lors de la phase de croissance de la vigne dès que la fraction d'eau disponible (calculée par le modèle de bilan hydrique WALIS) passe en dessous de 0,1. Cette valeur seuil peut être modulée à la hausse en fonction de la stratégie du viticulteur et des propriétés biophysiques de la parcelle (proximité d'un cours d'eau, situation en haut ou bas de pente, exposition).

Les besoins d'irrigation sont donc très variables en fonction des sites et des caractéristiques des parcelles. Une synthèse réalisée sur trois zones de production de la bodega Alta Vista illustre cette importante variabilité (figure 10). Le nombre de jours d'irrigation est généralement sousestimé par le modèle car il ne tient pas compte de la durée des apports qui se font majoritairement sous forme gravitaire. Certains apports peuvent s'étendre sur plusieurs jours alors que le modèle considère que la réserve en eau du sol est à son maximum dès le déclenchement du processus d'irrigation. 


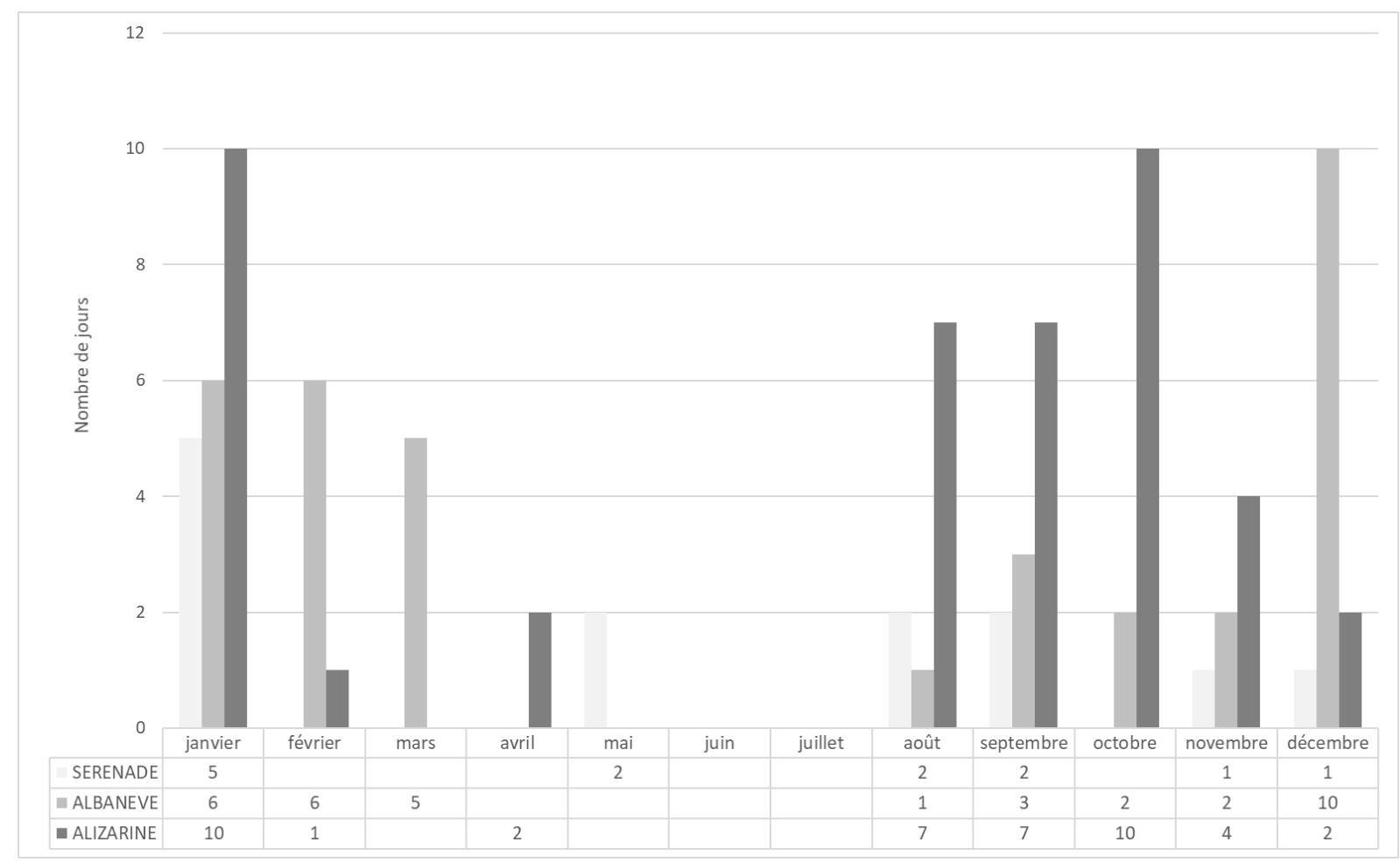

Figure 10 : Nombre de jours d'irrigation simulés par le modèle dans trois zones de production en 2014 (valeurs données pour une parcelle test de chaque zone) / Number of irrigation days simulated by the model in three production areas in 2014 (values are given for a test plot for each area)

Malgré ces limites, on note la très forte dépendance à l'irrigation des zones de production. Certaines parcelles comme celles d'Alizarine peuvent être irriguées jusqu'à 10 jours par mois. D'autres comme celles de la zone de production de Sérénade sont moins consommatrices. La répartition des apports en eau dépend de la capacité de rétention des sols qui sont un des paramètres essentiels du modèle de bilan hydrique. Le déclenchement de l'action d'irrigation par l'agent "Viticulteurs" intervient dès que le stress hydrique devient fort, sans qu'il n'y ait d'anticipation par l'utilisation d'action préventive comme le travail du sol ou le mulching sous le rang (ces actions restent rarement pratiquées dans la région de Mendoza).

Ce constat est à mettre en relation avec les projections des modèles climatiques régionalisés qui simulent une augmentation de $1,5^{\circ} \mathrm{C}$ à $3^{\circ} \mathrm{C}$ de la température moyenne à $1^{\prime}$ horizon 2100 , suivant le scenario retenu et une diminution des précipitations hivernales dans les Andes entre -1 et 4mm/jour (Chou et al., 2014 ; Barros et al., 2015). Comme précédemment évoqué, le lien avec ces données reste difficile à établir à l'échelle parcellaire, en raison de la forte variabilité locale des conditions climatiques. Ces projections posent néanmoins la question de la durabilité des systèmes de production actuels à moyen terme. Il est ainsi probable que des solutions alternatives permettant de retarder le stress hydrique soient mises en œuvre à moyen terme car 
les droits d'accès à l'eau sont de plus en plus contrôlés dans un contexte de forte augmentation des besoins.

\section{CONCLUSION ET PERSPECTIVES}

La démarche de modélisation présentée dans cet article propose une approche globale visant à simuler le déroulement des activités viticoles sous contraintes climatiques. Abordée via le développement d'un modèle multi-agents chargé de modéliser les interactions entre les différentes composantes de la viticulture, la problématique de l'impact de la variabilité des conditions climatiques sur les stratégies de production viticole a été plus spécifiquement ciblée.

Les premiers résultats obtenus, même s'ils restent partiels, montrent que le modèle DAHUVigne est en mesure de restituer la dynamique de la vigne et de simuler des itinéraires de conduites agronomiques en lien avec la variabilité des conditions climatiques. Cette étape est cruciale car elle constitue un préalable indispensable à l'analyse des stratégies de production dans le contexte du changement climatique.

A l'issue de cette première phase d'implémentation, de nombreux développements complémentaires (dont certains sont toujours en cours) ont été engagés. Ils concernent principalement des améliorations du code de calcul du modèle, qui nécessite d'être étoffé pour évaluer les implications de certains outils d'adaptation sur la dynamique de la vigne. Sur le plan technique, cela revient à introduire des boucles de rétroaction au sein du modèle pour simuler les implications des choix de conduites agronomiques sur les caractéristiques de la vigne (vigueur, précocité, résistance aux pathogènes...). Cette démarche permettrait notamment de modéliser l'influence d'actions sur le contrôle du bilan hydrique de la vigne et d'anticiper certaines actions et outils d'adaptation. Concernant l'irrigation, le développement d'une fonction de remplissage du réservoir du sol pourrait permettre d'évaluer les apports en nombre d'heures.

Les collaborations engagées avec les viticulteurs posent également la question de l'appropriation d'une telle démarche de modélisation dans un contexte où les savoir-faire et l'expérience restent les meilleurs outils d'adaptation à la variabilité du climat. Les échanges avec les professionnels sont indispensables pour valider et améliorer le modèle mais plus encore pour construire des scénarios tangibles concernant l'adaptation des pratiques au changement climatique. Ils permettent également de crédibiliser la démarche auprès de la profession viticole, ce qui constitue un préalable indispensable à la conduite de simulations participatives et prospectives. 
Si dans le contexte de la collaboration avec la bodega Alta Vista, les échanges ont pu être réalisés à toutes les phases de la modélisation en raison d'une attitude très volontariste et proactive du viticulteur, une diffusion plus large de ce type d'initiative au sein des structures viticoles argentines est plus complexe à réaliser. Cette difficulté s'explique par la diversité des perceptions du changement climatique et des stratégies d'adaptation envisagées. Certains viticulteurs anticipent par exemple une limitation de l'accès à l'irrigation dans la région de Mendoza et délocalisent leur vignoble plus au sud.

La démarche présentée dans cet article se poursuit actuellement au sein du projet LIFEADVICLIM qui étend l'approche menée en Argentine à plusieurs sites viticoles en Europe (en France, en Roumanie, en Allemagne, en Espagne et en Angleterre).

\section{Bibliographie}

Ashenfelter, O., Storchmann, K., 2016. Climate change and wine: A review of the economic implications. Journal of Wine Economics, 11(1), 105-138. doi:10.1017/jwe.2016.5

Baggiolini M., 1952. Les stades repères dans le développement annuel de la vigne et leur utilisation pratique. Revue romande d'Agriculture et d'Arboriculture 8 (1), p. 4-6.

Barros, V. R., Boninsegna, J. A., Camilloni, I. A., Chidiak, M., Magrín, G. O. et Rusticucci, M., 2015. Climate change in Argentina : Trends, projections, impacts and adaptation. Wiley Interdisciplinary Reviews: Climate Change, 6(2): p.151-169.

Battaglini, A., Barbeau G., Bindi M., Badeck F. W., 2009. European winegrowers' perceptions of climate change impact and options for adaptation. Regional Environ. Change 9, 61-73. doi:10.1007/s10113-008-0053-9

Barbeau C., Barbeau G., Joannon A., 2014. Analyzing the sensitivity of viticultural practices to weather variability in a climate change perspective: an application to workable-day modelling, Journal International des Sciences de la Vigne et du Vin, ${ }^{\circ} 48(2)$, pp. 141-152.

Barreteau O., Bousquet F., Etienne M., Souchère V., D’Aquino P., 2010. La modélisationd'accompagnement: une méthode de recherche participative et adaptative. In La modélisation d'accompagnement: une démarche d'appui au développement durable, Editions Quae, Update Sciences \& Technologies, pp. 21-46.

Canziani, P. O. et Scarel, E. A. (2010). South American Viticulture, Wine Production and Climate Change, UCA, $18 \mathrm{p}$.

Celette F., Ripoche A., Gary C., 2010. WaLIS - A simple model to simulate water partitioning in a crop association : The example of an intercropped vineyard. Agricultural Water Management, 97(11), 1749-1759. Doi : 10.1016/j.agwat.2010.06.008

Duchêne E, Schneider C., 2005. Grapevine and climatic changes: a glance at the situation in Alsace. Agron Sustain Dev 25, 93-99. doi: 10.1051/agro:2004057

Chou, S. C., Lyra, A., Mourão, C., Dereczynski, C., Pilotto, I., Gomes, J., Bustamante, J., Tavares, P., Silva, A., Rodrigues, D. et al., 2014. Assessment of climate change over South America under RCP 4.5 and 8.5 downscaling scenarios. American Journal of Climate Change, 3(5): p. 512-525.

Edmonds B., Le Page C., Bithell M., Chattoe-Brown E., Grimm V., Meyer R., Montañola-Sales C., Ormerod 
P., Root H., Squazzoni F., 2019. Different Modelling Purposes Journal of Artificial Societies and Social Simulation 22 (3) 6, doi: 10.18564/jasss.3993

Fraga H, Malheiro A., Moutinho-Pereira J, Santos J., 2012. An overview of climate change impacts on European viticulture. Food Energy Secur 1(2), p. 94-110. doi:10.1002/fes3.14

González, G., Nazrala, J., Beltrán, M., Navarro, A., De Borbón, L., Senatra, L., Albornoz, L., Hidalgo, A., et al., 2009. Caracterización de uvas para vinificar en diferentes regiones de Mendoza (Argentina). Revista de la Facultad de Ciencias Agrarias, 41(1): p. 165-175.

Grimm V., Berger U., Bastiansen F., Eliassen S., Ginot V., Giske J., Goss-Custard J., Grand T., Heinz S.K., Huse G., Huth A., Jepsen J.U., Jorgensen C., Mooij W.M., Muller B., Pe'er G., Piou C., Railsback S. F., Rob-Bins A.M., Robbins M.M., Rossmanith E., Ruger N., Strand E., Souissi S., Stillman R.A., Vabo R., Visser U., Deangelis D.L., 2006. “A standard protocol for de-scribing individual-based and agent-based models”,Ecological Modelling, vol. $198, \mathrm{n}^{\circ} 1-2$, pp. 115-126.

Grimm V., Berger U., Deangelis D.L., Polhill J., Giske J., Railsback S., 2010. “The ODD protocol: A review and first update", Ecological Modelling, vol. 221, pp. 2760-2768.

Griset, P., Laborie, L., 2016. Introduction. De qui, de quoi, malbec est-il le nom? Revista Iberoamericana de Viticultura, Agroindustria y Ruralidad, vol. 3, núm. 7, Universidad de Santiago de Chile, 11 p.

Huglin P., Schneider, C., 1998. Biologie et écologie de la vigne, Lavoisier Tec et Doc, Paris, 370 p.

Jones G.V., 2007. Climate Change: Observations, Projections, and General Implications for Viticulture and Wine Production, Practical Winery and Vineyard, p. 44-64.

Jones G.V., Davis R.E., 2000. Using a synoptic climatological approach to understand climate-viticulture relationships, International Journal of Climatology, n²0(8), p. 813-837.

Jones, G.V., 2015. A Global Perspective in Grapevine in a Changing Environment. In Grapevines in a Changing Environment: A Molecular and Ecophysiological Perspective. Edited by Hernâni Gerós, Maria Manuela Chaves, Hipolito Medrano Gil, Serge Delrot. Wiley-Blackwell, 400 p.

Keller, M., 2010. Managing grapevines to optimise fruit development in a challenging environment: A climate change primer for viticulturists. Aust. J. Grape Wine Res., 16, 56-69. doi:10.1111/j.1755-0238.2009.00077

Lavie, E., 2009. Activités Anthropiques et Qualité de l'eau Dans l'oasis de Mendoza (Argentine) : Diagnostic, Enjeux et Durabilité. Thèse de doctorat, Université Bordeaux 3, 383 p.

Loussert P., 2017. Caractérisation de la viticulture irriguée par télédétection en contexte de changement climatique : application aux vignobles de la province de Mendoza en Argentine. Thèse de géographie, Univeristé de Rennes 2, 226 p.

Mosedale, J. R., Abernethy, K. E., Smart, R. E., Wilson, R. J., Maclean, I., 2016. Climate change impacts and adaptive strategies: lessons from the grapevine. Global Change Biology, 22(11), 3814-3828. doi: $10.1111 /$ gcb. 13406

Ojeda H., Saurin N., 2014. L’irrigation de précision de la vigne : méthodes, outils et stratégies pour maximiser la qualité et les rendements de la vendange en économisant de l'eau. Innovations Agronomiques 38, p. 97-108.

OIV, 2019. Note de conjoncture mondiale : situation du secteur en 2018, 16 p.

Ollat, N., Touzard, J. M., \& van Leeuwen, C., 2016. Climate change impacts and adaptations: New challenges for the wine industry. Journal of Wine Economics, 11(01), p. 139-149. doi: 10.1017/jwe.2016.3 
Parker A., Garcia De Cortazar Atauri I., Chuine I., Barbeau G., Bois B., Boursiquot J.-M., Cahurel J.-Y., Claverie M., Dufourcq T., Geny L., Guimberteau G., Hofmann R. W., Jacquet O., Lacombe T., Monamy C., Ojeda H., Panigai L., Payan J.-C., Lovelle B. R., Rouchaud E., Schneider C., Spring J.-L., Storchi P., Tomasi D., Trambouze W., Trought M., Van Leeuwen C., 2013. Classification of varieties for their timing of flowering and veraison using a modelling approach: A case study for the grapevine species Vitis vinifera L. Agricultural and Forest Meteorology, 180, p. 249-264. doi:10.1016/j.agrformet.2013.06.005

Quénol H., Bonnardot V., 2014: A multi-scale climatic analysis of viticultural terroirs in the context of climate change: the "TERADCLIM" project. International Journal of Vine and Wine Sciences, p. 23-32.

Sklar E., 2011. "NetLogo, a multi-agent simulation environment." Artificial Life, 13(3): p 303-311.

Schultz H.R., Jones G.V., 2010. Climate induced historic and future changes in viticulture. J Wine Res 21, p. 137-145. doi: 10.1080/09571264.2010.530098

Tissot C., Le Tixerant M., Rouan M., 2005. Le simulateur DAHU : une plate-forme de modélisation des activités humaines en zone côtière, Norois, ${ }^{\circ} 196$, p. 125-135.

Tissot C., Neethling E., Rouan M., Barbeau G., Quénol H.., Le Coq C., 2017. Modeling Environmental Impacts on Viticultural Ecosystems: A First Case Study in a Regulated Wine Producing Area, International Journal of Agricultural and Environmental Information Systems (IJAEIS), n8(3), p. 1-20.

Tissot C., 2018. Modélisation du déroulement d'activités anthropiques sous contraintes spatio-temporelles, HDR de géographie, Université de Bretagne Occidentale, 109 p.

Tonietto J., Carbonneau A., 2004. A multicriteria climatic classification system for grape-growing regions worldwide. Agricultural and Forest Meteorology, 124/1-2, p. 81-97.

Van Leeuwen C., Friant P., Chone X., Tregoat O., Koundouras S., Dubourdieu D., 2004. The influence of climate, soil and cultivar on terroir. Am. J. Enol. Vitic., 55, $n^{\circ} 3,207-217$.

Van Leeuwen C., Garnier C., Agut C., Baculat B., Barbeau G., Besnard E., Bois B., Boursiquot J.., Chuine I.., Dessup T., 2008. Heat requirements for grapevine varieties is essential information to adapt plant material in a changing climate, VII th International terroir Congress (Nyon), pp 222-227

Van Leeuwen, C., Darriet, P., 2016. The impact of climate change on viticulture and wine quality. Journal of Wine Economics, 11(1), p. 150-167. doi:10.1017/jwe.2015.21

Vigil, A., Ruiz de Peña y Lillo, A. et Villa, H., 2003. Nuevas estructuras de la vitivinicultura argentina. In DíasÁlavrez, J. et Laureano, O., éditeurs : XIX.C Red Ibero-Americana de Vitivinicultura. A Vitivinicultura Nos Países Iberoamericanos : Impacto Económico, Social e Técnico-Científico. Instituto Superior de Agronomia de Portugal. 